Proceedings

\title{
Dynamic Displacements Measurement Employing Fringe Projection and Digital Image Correlation ${ }^{+}$
}

\author{
Luis Felipe-Sesé ${ }^{1, *}$, Ángel Molina-Viedma ${ }^{2}$, Elías López-Alba ${ }^{2}$ and Francisco Díaz ${ }^{2}$ \\ 1 Departamento de Ingeniería Mecánica y Minera, Campus Científico Tecnológico de Linares, \\ Universidad de Jaén, 23700 Linares, Spain \\ 2 Departamento de Ingeniería Mecánica y Minera, Campus Las Lagunillas Universidad de Jaén, \\ 23071 Jaén, Spain; ajmolina@ujaen.es (Á.M.-V.); elalba@ujaen.es (E.L.-A.); fdiaz@ujaen.es (F.D.) \\ * Correspondence: lfelipe@ujaen.es; Tel.: +34-953-64-85-74 \\ + Presented at the 18th International Conference on Experimental Mechanics (ICEM18), Brussels, Belgium, \\ 1-5 July 2018.
}

Published: 18 June 2018

\begin{abstract}
A combination of Fringe Projection (FP) and 2D Digital Image Correlation (2D DIC) using a single camera has been employed to simultaneously measure in-plane and out-of-plane displacements during different dynamic events such an impact and a vibration analysis. This approach has been adopted in the past by several authors, including Mares et al. who highlighted that if a telecentric lens are not employed the combination of both techniques is not straightforward since the in-plane displacements measured with 2D DIC are sensitive to the out-of-plane displacements. Thus, in-plane displacements measured using 2D DIC should be corrected using the out-of-plane displacements inferred using the FP technique. In the current work, an easy method for in-plane displacement correction using Fringe Projection is adopted when measuring the 3D displacements during an impact on an aluminum plate and the vibration of a composite component which have not been studied by other authors previously.
\end{abstract}

Keywords: digital image correlation; Fringe Projection; dynamic displacement measurements

\section{Introduction}

The application of optical full-field techniques in experimental mechanics is increasing notoriously in recent years thanks to the improvements of technology in cameras and image processing. Especially important are their possibilities in dynamic analysis such as vibration analysis and impact analysis. Full field results of these optical techniques could help in the increasing of component life, health monitoring, numerical method updating or the optimization of design among others. One of the most spread techniques is Digital Image Correlation 2D or 3D which offers displacement or strain fields up to thousands of samples per second employing one or two high speed cameras respectively.

In this work, an alternative to this DIC 3D Technique has been applied. Specifically, a combination of Fringe Projection (FP) [1] and 2D Digital Image Correlation (2D DIC) [2] using a single camera has been employed to simultaneously measure in-plane and out-of-plane displacements during different dynamic events such an impact and vibration analysis. This approach has been adopted in the past by several authors, including Mares et al. [3] who highlighted that if a telecentric lens is not employed the combination of both techniques is not straightforward since the in-plane displacements measured with 2D DIC are sensitive to the out-of-plane displacements experienced by the object during deformation. Thus, in-plane displacements measured using 2D DIC should be corrected using the out-of-plane displacements inferred using the FP technique. 
In the current work, an easy method for in-plane displacement correction previously developed by the authors [4] using FP and 2D DIC is adopted when measuring the 3D displacements. The aim is to highlight the potential of this combination of optical techniques in the analysis of dynamic testing when expensive high speed cameras are employed. Specifically, for illustration purposes, an impact test on an aluminum plate and a vibration test on a composite cantilever beam were performed.

\section{Background}

\section{Technique FP + 2D DIC}

The analysis of dynamic mechanics events will usually imply large in-plane displacements, and, also, not negligible out-of-plane displacements. Thus, for accurate full field measurement of the specimen deformation it is required an experimental technique that accounts for both in-plane and out-of-plane displacements simultaneously. Two experimental techniques that allow performing this kind of measurements are 3D-DIC [5] and now, the combination of FP and 2D-DIC also satisfy this requirement $[4,6,7]$.

To implement digital image correlation (2D- as well as 3D-DIC), the specimen surface has to be previously prepared by spraying it with a random speckle pattern $[2,5]$. In addition for fringe projection [8,9], vertical fringes have to be projected on the specimen surface with non-cero incidence angle $(\alpha)$ respect to the optical axis (O.A.) as shown in Figure 1. Thus, when the surface is deformed, both speckle and projected fringes moved accordingly. 2D-DIC computes speckle displacements in a plane perpendicular to the optical axis while FP computes the surface displacement along the optical axis from the lateral shifting of the projected fringes. Therefore, when FP and 2D-DIC are performed at the same time, both patterns have to be conveniently separated to integrate both techniques simultaneously. Usually, speckle images are processed with a commercial software which compiles correlation algorithms with subpixel accuracy [10], while fringe images requires a phase extraction procedure [11,12] and unwrapping process [13].

The adopted method for the simultaneous measurement of the in- and out-of plane displacements employs a conventional colour LCD projector and a colour (i.e., RGB) digital camera [14]. The specimen was painted with red speckle over white background and blue-white fringes were projected with a sinusoidal intensity profile.

As previously mentioned, if a telecentric lens is not employed, the in-plane displacements are distorted and they must be corrected using the out-of-plane information. The correction method adopted [4] obtains real in-plane displacement using the out-of-plane displacement obtained with FP. It is based on a pin-hole model with the assumption that the relative small out-of-plane displacement does not affect the image quality due to defocusing effect. This method relates for each pixel the corrected in-plane displacements $(\Delta x$ and $\Delta y)$ with the uncorrected ones $\left(\Delta x_{C C D}, \Delta y_{C C D}\right)$ directly obtained with 2D-DIC and the out-of-plane distance $(\Delta z)$ measured with FP:

Where $\left(x_{1}, C C D, y_{1}, C C D\right)$ and $\left(x_{2}, C C D, y_{2}, C C D\right)$ are the initial and final position of a displaced pixel at the element surface, $\Delta z_{1}$ and $\Delta z_{2}$ are the corresponding out-of-plane displacement at that pixel, $z_{0}$ is the distance between the reference surface and the optical centre of the camera lens and $L$ is the inverse of the lateral magnification at $z$.

$$
\left\{\begin{array}{l}
\Delta x=L\left[\Delta x_{C C D}-\left(x_{2, C C D} \frac{\Delta z_{2}}{z_{0}}-x_{1, C C D} \frac{\Delta z_{1}}{z_{0}}\right)\right] \\
\Delta y=L\left[\Delta y_{C C D}-\left(y_{2, C C D} \frac{\Delta z_{2}}{z_{0}}-y_{1, C C D} \frac{\Delta z_{1}}{z_{0}}\right)\right]
\end{array}\right.
$$

To perform the correction using Equation (1) the following parameters are required: the distance $z_{0}$, the relation $L$, and the intersection of the optical axis with the CCD plane (i.e., the centre of the CCD) from which the pixel positions are referenced. In addition, FP requires the fringe constant, $K_{F P}$, that relates the amount of phase shifting, $\Delta \phi$, produced by the fringe displacement due to the $z$ displacement. All these parameters can be obtained following a calibration process [7]. 


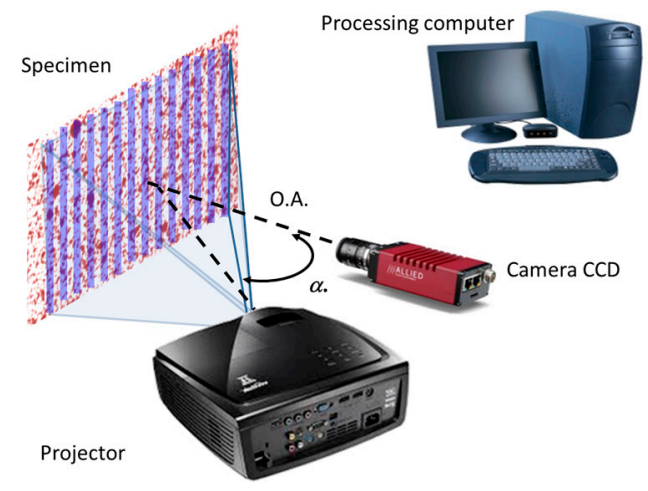

Figure 1. Schematic set-up for FP+ 2D DIC Technique.

\section{Apparatus and Methods}

\subsection{Impact Test}

The impact test was conducted on a $150 \times 150 \times 3 \mathrm{~mm}^{3} 1050$ aluminium plate. The plate was completely clamped in its four edges having a free area of $120 \times 120 \mathrm{~mm}^{2}$. The test consisted of a dropweight test, where a $4 \mathrm{~mm}$ radius hemispheric steel indenter (considered as completely rigid) impacted the specimen. A mass of $7.21 \mathrm{~kg}$ was positioned at a $141.4 \mathrm{~mm}$ height and released to generate impact energy of $10 \mathrm{~J}$. For measurement purposes, the camera observes the rear part of the specimen by the use of a $45^{\circ}$ mirror, as shown in Figure 2. Impact force was registered during the event with a Kistler $20 \mathrm{kN}$ Load Cell. Images were captured employing a Photron SA3 Cam recording at $3000 \mathrm{fps}$ and a Epson $\times 11$ projector projected the oblique blue fringe pattern.

After the test, colour images were decomposed into a speckle and fringe pattern images. Speckle images were processed employing VIC 2D (with facet size of 35px and 3px spacing) software from Correlated Solutions. Fringe images were processed using a Fourier Profilometry [9] with a quality guided unwrapping process [13].

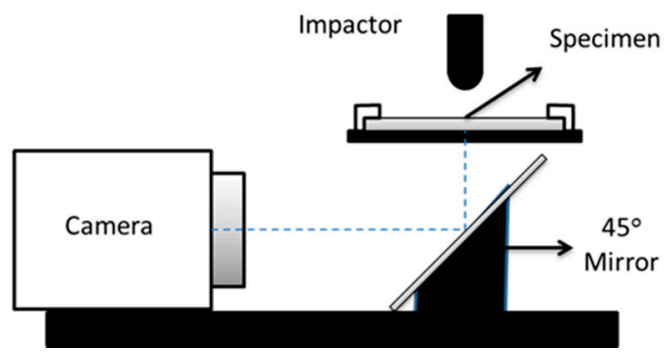

Figure 2. Scheme of the experimental set-up for impact test on aluminium plate.

\subsection{Vibration Test Test}

In this test a composite cantilever bean was subjected to a modal analysis and results were compared with those obtained with a numerical simulation.

The tested specimens consisted on an $250 \times 25 \times 1.99 \mathrm{~mm}$ (ASTM D-3039) rectangles of glass fiber with an orientation of the fibers of $[\mathrm{M}, 0, \mathrm{M}, 0]$, being $\mathrm{M}$ an aleatory distribution and manufactured following the hand lay procedure. The matrix was an Orthophthalic Polyester Resin. The fabrication followed the hand lay-up procedure to obtain a total mass of the specimen of $20.69 \mathrm{gr}$.

A previous tension test was performed over other similar specimen in order to obtain the elastic parameters of the specimen. Additionally, a previous random vibration test was performed in order to detect the resonance frequencies employing an accelerometer placed close to the clamping area to minimize the additional mass effect (Figure 3c). 
Results were employed to achieve a Finite Element Method of the specimen. Abaqus 6.14 was employed to perform a Composite Lay-up model with 250 elements. Additional mass due to accelerometer was also taken into account in the FEM model.

The set up for the vibration test employed similar main elements as impact test, i.e., an Epson EB-W32 projector and a Photron SA3 Camera recording up to 5000 frames per second as observed in Figure 3. The specimen was excited through a Data Physics GW-V20/PA30E shaker (Figure 3b). Similar imagen processing was employed.
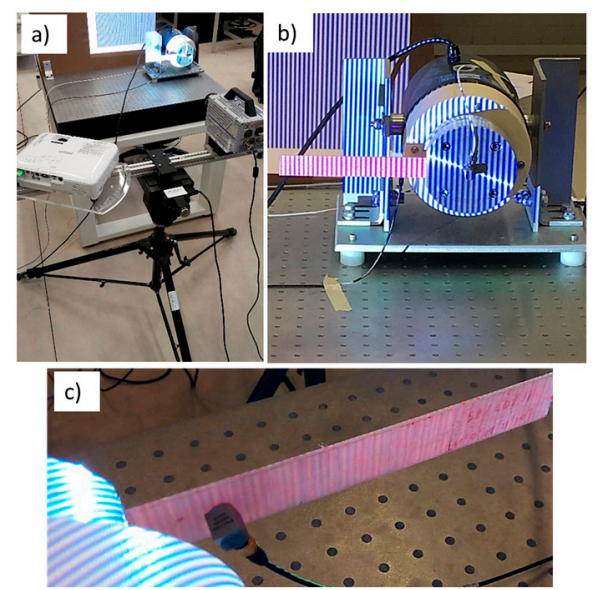

Figure 3. Experimental set-up for vibration test. (a) General view (b) Detail of the composite specimen clamped on the shaker (c) Detail of accelerometer.

\section{Results}

In Figure 4 it is presented the maximum y-direction (Figure $4 \mathrm{~A}$ ) and out-of-plane in z-direction (Figure 4B) displacement measured during the impact test. Results show a clear symmetric behavior. Z-displacements present some noise due to speckle pattern but it is noteworthy the good level of accuracy also in Y-displacement since displacement below $0.1 \mathrm{~mm}$ could be observed clearly, which is below the $\mathrm{mm} /$ pixel relation for this test. These Y-displacement data will

Respect to vibration test, Table 1 presents the results of the resonance frequencies associated to the first three bending modes of resonance. As observed, differences in the frequencies increase from a reasonable $12 \%$ in the first mode to a more notorious $26 \%$ in the third mode. Taking into account that the specimen was made following the hand lay out procedure, those differences are expected. This procedure could lead to have some air inclusions with are difficult to model and will affect to the final behaviour.
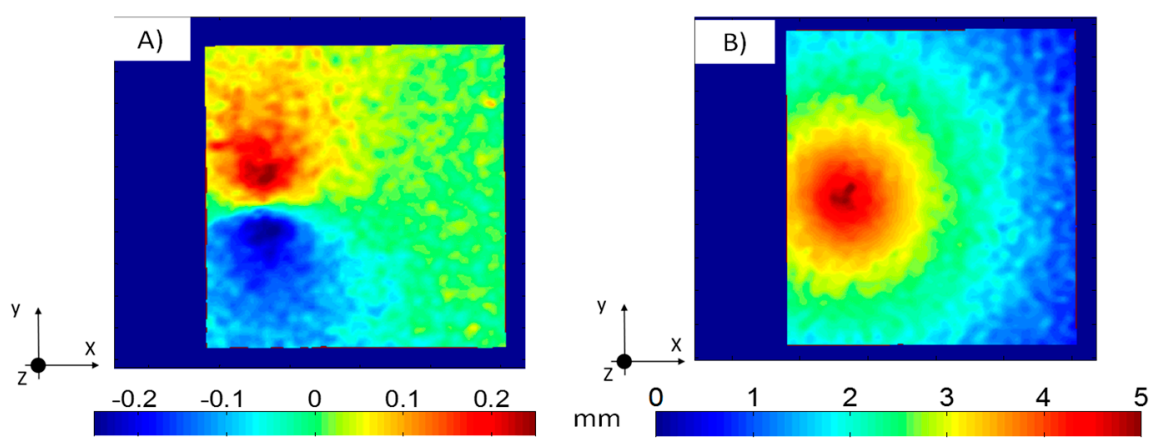

Figure 4. (A) Y-Direction and (B) Z-Direction displacement maps occurring at maximum displacement instant during test. 
Table 1. Results of frequencies associated to the first three resonance modes of the composite cantilever beam.

\begin{tabular}{ccc}
\hline Mode & FEM & Experimental \\
\hline 1 & $37.3 \mathrm{~Hz}$ & $33.1 \mathrm{~Hz}$ \\
2 & $234 \mathrm{~Hz}$ & $206 \mathrm{~Hz}$ \\
3 & $650 \mathrm{~Hz}$ & $515 \mathrm{~Hz}$ \\
\hline
\end{tabular}

Figure 5 presents the displacement maps measured and simulated with FEM. As observed, Modes 1 and 2 shows a really good agreement, in concordance with the small difference in their frequencies. Nevertheless, experimental results of mode 3 show some longitudinal asymmetry, possibly due to some heterogenic stiffness distribution. Additionally, it is observed relatively large displacement where the accelerometer was placed, which enhances the mass effect. These two effects must be the reason of the big difference in frequency.

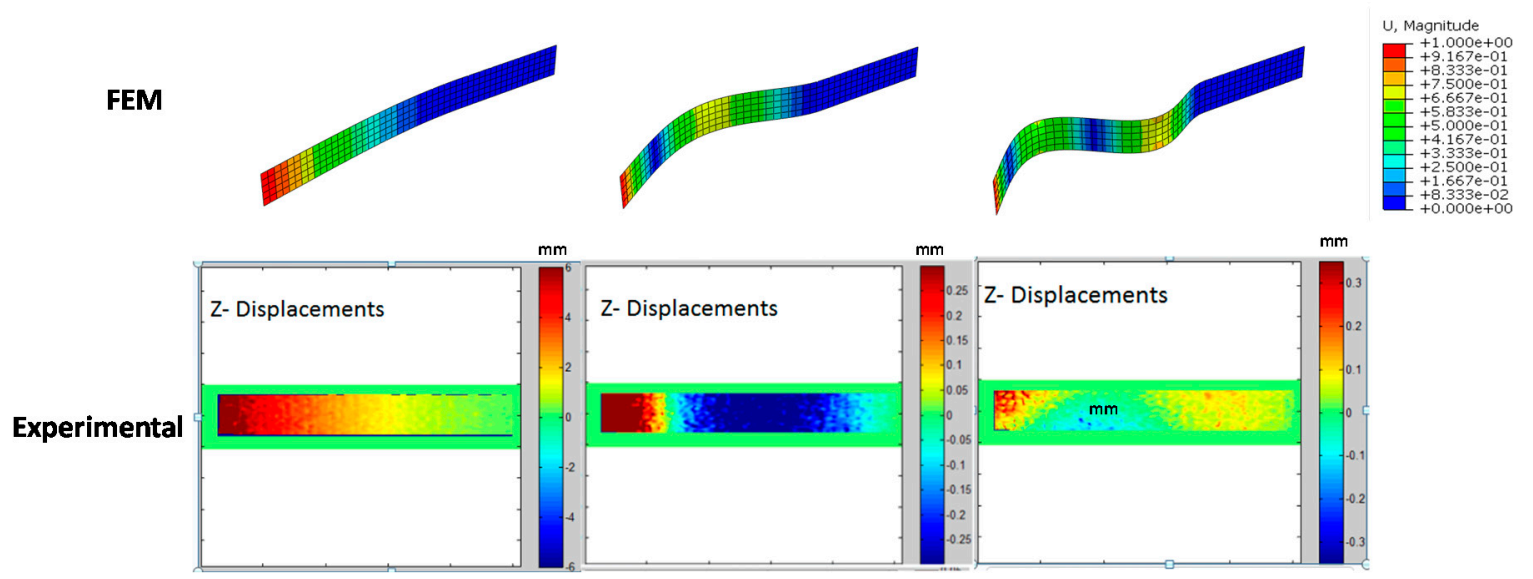

Figure 5. Modal test results of Finite element Method (left) and FP+2D-DIC technique (right).

\section{Conclusions}

As observed, FP+2D-DIC has been applied to interesting dynamic examples highlighting its possibilities and the possibility of a further mechanical analysis. The results could be compared with those obtained with 3D-DIC although some noise could be present in the displacement results. Nonetheless, it is important to notice that the reduction in the cost of the set-up is important. Additionally, there is a simplification of the set-up, which was important in the impact test where the space for the optical layout was limited. It should be consider as a real alternative to 3D-DIC in dynamic mechanical testing.

Conflicts of Interest: The authors declare no conflicts of interest.

\section{References}

1. Su, X.; Chen, W.; Xiangyu Su, W.C. Fourier transform profilometry a review.pdf. Opt. Lasers Eng. 2001, 35, 263-284.

2. Pan, B.; Qian, K.; Xie, H.; Asundi, A. Two-dimensional digital image correlation for in-plane displacement and strain measurement: A review. Meas. Sci. Technol. 2009, 20, 1-17.

3. Mares, C.; Barrientos, B.; Blanco, A. Measurement of transient deformation by color encoding. Opt. Express 2011, 19, 25712-25722.

4. Felipe-Sesé, L.; Siegmann, P.; Díaz, F.A.; Patterson, E.A. Simultaneous in-and-out-of-plane displacement measurements using fringe projection and digital image correlation. Opt. Lasers Eng. 2014, 52, 66-74.

5. Sutton, M.A.; Orteu, J.-J.; Schreider, H. Image Correlation for Shape, Motion and Deformation Measurements Basic Concepst, Theory and Applications; XXXIII (2); Springer: Boston, MA, USA, 2009.

6. Felipe-Sesé, L.; López-Alba, E.; Siegmann, P.; Díaz, F.A. Integration of fringe projection and twodimensional digital image correlation for three-dimensional displacements measurements. Opt. Eng. 2016, 
55, 121711.

7. Felipe-Sesé, L.; Siegmann, P.; Díaz, F.A.; Patterson, E.A. Integrating fringe projection and digital image correlation for high-quality measurements of shape changes. Opt. Eng. 2014, 53, 044106.

8. Heredia-Ortiz, M.; Patterson, E.A. On the Industrial Applications of Moire and Fringe Projection Techniques. Strain 2003, 39, 95-100.

9. Takeda, M.; Ina, H.; Kobayashi, S. Fourier-transform method of fringe-pattern analysis for computer-based topography and interferometry. J. Opt. Soc. Am. 1982, 72, 156-160.

10. Schreier, H.; Orteu, J.-J.; Sutton, M.A. Image Correlation for Shape, Motion and Deformation Measurements; Springer: Boston, MA, USA, 2009.

11. Ortiz, M.H.; Patterson, E.A. Location and Shape Measurement Using a Portable Fringe Projection System. Exp. Mech. 2005, 45, 197-204.

12. Huntley, J.M. Automated fringe pattern analysis in experimental mechanics: A review. J. Strain Anal. Eng. Des. 1998, 33, 105-125.

13. Ghiglia, D.; Pritt, M. Two-Dimensional Phase Unwrapping: Theory, Algorithms, and Software; Wiley: New York, NY, USA, 1998.

14. Siegmann, P.; Álvarez-Fernández, V.; Díaz-Garrido, F.; Patterson, E. A simultaneous in- and out-of-plane displacement measurement method. Opt. Lett. 2011, 36, 10-12.

(C) 2018 by the authors. Licensee MDPI, Basel, Switzerland. This article is an open access article distributed under the terms and conditions of the Creative Commons Attribution (CC BY) license (http://creativecommons.org/licenses/by/4.0/). 